\title{
Operational fault states in railways
}

\author{
Andreas Schöbel • Thomas Maly
}

Received: 21 June 2010 / Accepted: 12 December 2011 / Published online: 18 January 2012

(C) The Author(s) 2012. This article is published with open access at SpringerLink.com

\begin{abstract}
Fault states In railway systems, there are many different fault states which can occur during operation. For safety reasons, these well known fault states have to be prevented by train monitoring. But fault states may also have dependencies among each other, which are not sufficiently known yet or used for fault state prevention.

Approach of systematic description Thus, in a first step an abstract approach has been developed for the description of dependencies. Based on this and as systematic description of all relevant states and their dependencies a fault state matrix has been created, which is considered in this paper.
\end{abstract}

Keywords Fault states $\cdot$ Railway operation $\cdot$ Safety $\cdot$ Causeconsequence-dependency

\section{Introduction}

This paper deals with the results from a national founded project in Austria [1]. Aim of this project was to describe the complex dependencies in the field of railway safety from a technical point of view in a simplified way for practical usage of infrastructure managers and railway undertakings in their

\footnotetext{
A. Schöbel $\cdot$ T. Maly $(\bowtie)$

Institute of Transportation, Research Center for Railway

Engineering, Traffic Economics and Ropeways,

Vienna University of Technology,

Karlsplatz 13/230-2,

1040 Vienna, Austria

e-mail: thomas.maly@tuwien.ac.at

URL: www.eiba.tuwien.ac.at
}

\footnotetext{
A. Schöbel

e-mail: andreas.schoebel@tuwien.ac.at

URL: www.eiba.tuwien.ac.at
}

safety management system. The railway system has a lot of dependencies between different fault states, which can be described by an ontology based approach [2]. In general, faults state can lead to another fault state which might be worse than the one before. Thus, in an abstract approach fault states can be interpreted as causes and the resulting fault states can be interpreted as consequences (Fig. 1).

If there are no measures for recognition, the final consequence of many fault states is a derailment. Therefore it is necessary to prevent the long-lasting occurrence of all critical fault states. Because of the movement of the train, most of the relevant fault states can not be observed directly. Thus, suitable indicators have to be measured, which can be done by onboard or wayside monitoring systems [3].

\section{Fault state matrix}

\subsection{Fault states}

Based upon this abstract approach a matrix was created to point out the dependencies in a customer-friendly way (Fig. 2). For each fault state a description was developed accordingly to UIC dictionary an in relation to [4].

1. Derailment: at least one wheel is not running on the rail

2. Hot Box: axle-bearing exceed temperature threshold

3. Blocked Brake or Wheel: permanent braking of a wheel or an axle (rotating wheels and axles as well as blocking ones)

4. Faulty Flash Guard: missing flash guards or flash guards which are not able to prevent sparks touching vehicle

5. Faulty Elements of Brake System: faulty elements of the braking system (brake valve, brake leverage, etc.), which cause continuous braking

6. Broken Axle

7. Breakage of Stub Shaft

8. Broken Wheel 
Fig. 1 Abstract approach of causes and consequences

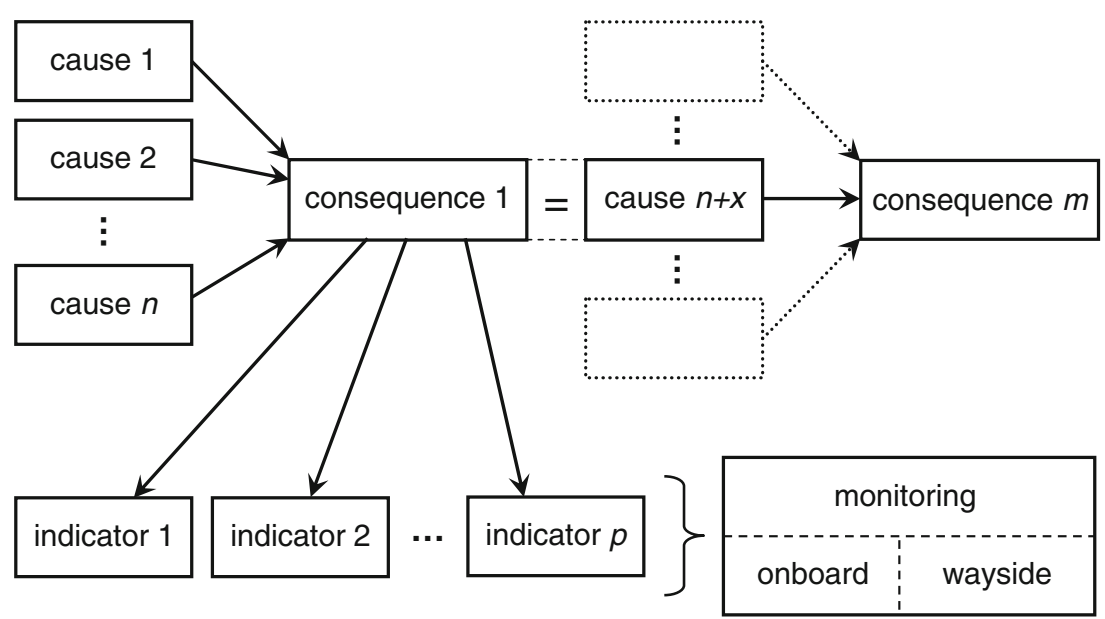

9. Faulty Running Surface/Wheel Spot: failure on the running surface (flat spot, pitting, rewelding, polygonal, etc.)

10. Faulty Flange of Wheel: too high wear (exceeding operational threshold) or flange with broken out parts
11. Faulty Suspension and-component: faulty suspension spring (wrong or different types installed, loosen or displaced spring band, cracks), broken suspension springs, broken or missing parts of suspension

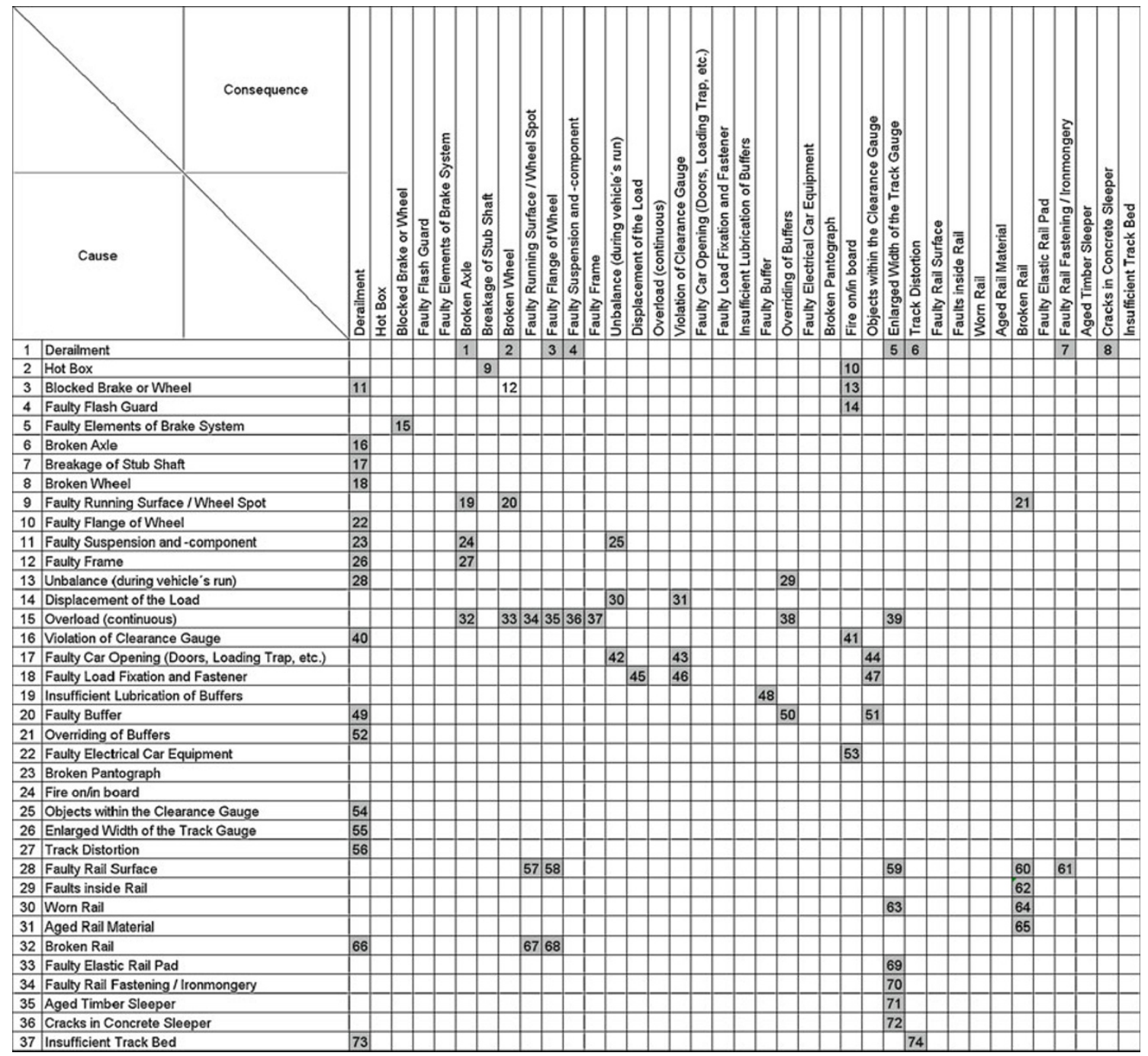

Fig. 2 Cause consequence matrix of fault states 
(suspension ring, elements of shock absorber), faulty suspension bracket (cracks, broken)

12. Faulty Frame: cracks and breakages on structural parts (mainly in the section of integration of crossbar in main frame), buckling of chassis, longitudinal buckling respectively forbidden deflection

13. Unbalance (during vehicle's run): different wheel load (left-right and/or front-rear) - only one single train journey considered in this context

14. Displacement of the Load

15. Overload (continuous): wheel, axle or car load exceeding operational threshold. In this context this fault state occurs during several train runs.

16. Violation of Clearance Gauge

17. Faulty Car Opening (Doors, Loading Trap, etc.): faulty car openings like doors, loading traps, sliding-walls, etc. which are not reliable closed or which are opening during the run

18. Faulty Load Fixation and Fastener: faulty belts, tarps, fixation net, stanchion

19. Insufficient Lubrication of Buffers

20. Faulty Buffer: dragged-in buffer disc, faulty spring element, loose or missing fixation elements, too high end play, cracked weldseam

21. Overriding of Buffers

22. Faulty Electrical Car Equipment: faulty electrical devices/systems e.g. air conditioner, heating, ventilation, light

23. Broken Pantograph: breakage of sliding contact

24. Fire on/in board

25. Objects within the Clearance Gauge: objects, which are lying on the superstructure and entering the clearance gauge

26. Variation of Width of the Track Gauge: exceeding or narrowing of the track gauge over allowed thresholds

27. Track Distortion: parallel, horizontal and/or vertical displacement of both rails and of sleepers

28. Faulty Rail Surface head checks, short-pitch corrugation, plastic yielding, etc.

29. Faults inside Rail: inclusions of material and welding failures

30. Worn Rail: lateral wear, longitudinal wear, etc.

31. Aged Rail Material: changed characteristics of rail material caused by fatigue or ageing

32. Broken Rail: all types of breakage (brittle fracture, comminuted fracture, etc.)

33. Faulty Elastic Rail Pad: faulty or missing elastic rail pad

34. Faulty Rail Fastening/Ironmongery: slack or broken rail fastening (bolts, clamps, etc.)

35. Aged Timber Sleeper

36. Cracks in Concrete Sleeper: longitudinal or lateral cracks
37. Insufficient Track Bed: wear of ballast, too less edge of ballast, reduction of bearing capacity due to insufficient drainage, base failure of an embankment, elevation due to poor frost resistance, etc.

\subsection{Transitions}

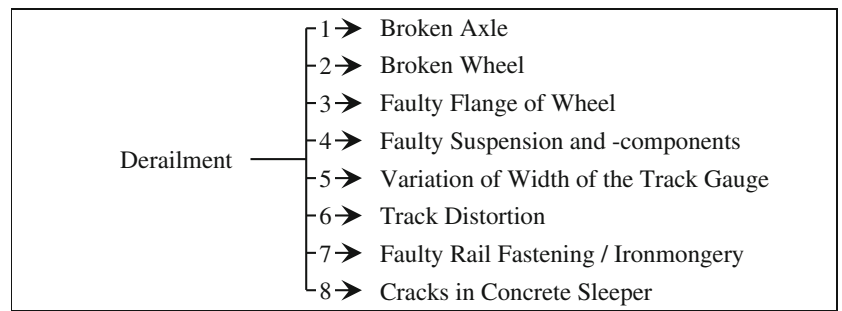

Description: A derailed wheel is influenced by high vertical acceleration impulses due to the alternation of sleepers and spaces between the sleepers. These conditions may cause damages on the wheel, axle and car suspension. Moreover the superstructure is stressed too and therefore also the elements of the superstructure (sleepers, fixations, track gauge) may get damaged.

Hot Box $\longrightarrow 9 \longrightarrow$ Breakage of Stub Shaft

Description: Material properties of boxes and axles are influenced by the high thermal stress which might cause a breakage of the stub shaft.

Hot Box $-10 \longrightarrow$ Fire on/in Board

Description: Due to thermal radiation respectively thermal conduction other components of a vehicle may be heated and get burned.

Blocked Brake or $-11 \longrightarrow$ Derailment

Wheel

Description: Continuous braking may lead under bad situations (acceleration, curve movement, bad combination of cars regarding their weight) due to additional longitudinal forces to a derailment of one axle. Moreover the thermal stress of wheel and axle may lead to a displacement of a wheel on the axle and thus to a reduced distance between the wheels. Therefore the risk of a derailment is rising caused by a play between rails and wheels.

Blocked Brake or $\longrightarrow 12 \longrightarrow$ Broken Wheel

Description: Shoe brakes transform due to friction the moving energy into heat. Thus there is a possibility of wheel breakage by thermal stress on the running surface. 


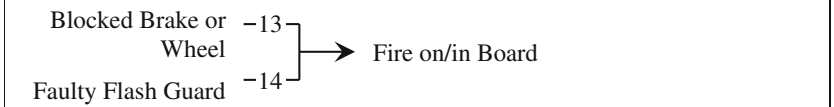

Description: While braking a train equipped with shoe brakes there is a possibility for the appearance of sparks, which might burn the deposit of dirt (oil, pollen). In combination with a faulty flash guard some sparks might burn the vehicle itself. Moreover the brake linings might burn in case of extensive use and cause therefore a fire on the whole vehicle.

Faulty Elements of $-15 \rightarrow$ Blocked Brake or Wheel

Description: Due to faulty components of the braking system a continuous braking can be caused (e.g. faulty control valve, bent brake leverage).

Broken Axle $-16 \longrightarrow$ Derailment

Description: If the rigid link between left and right wheel is missing, each of the wheels will be guided only by one axle box. This can lead to a derailment. Shaft $-17 \longrightarrow$ Derailment

Description: If the stub shaft breaks, the vertical forces cannot be assumed by the axle box anymore. Moreover the wheel can remove from the axle. Both may lead to a derailment.

Broken Wheel $-18 \rightarrow$ Derailment

Description: A broken wheel offers no guidance anymore. Therefore a derailment could take place.

$\begin{aligned} \text { Faulty Running } \\ \text { Surface / Wheel Spot }\end{aligned}-\left[\begin{array}{ll}19> & \text { Broken Axle } \\ 20> & \text { Broken Wheel } \\ 21> & \text { Broken Rail }\end{array}\right.$

Description: Faulty running surface leads to force peaks and causes therefore higher stress for wheel and rail.

$\begin{array}{r}\text { Faulty Flange of } \\ \text { Wheel }\end{array}-22 \longrightarrow$ Derailment

Description: If the wear of the wheel flange is getting higher the play between rails and wheels will be also higher, This may lead to a derailment under special operational conditions. If parts of the wheel flange are broken out, the guidance will be missed completely. This may lead to a derailment too.

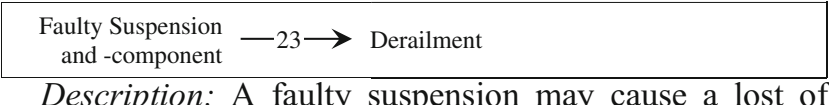
contact between wheel and rail. Due to the reduced absorption capability of faulty suspensions the vehicle body may oscillate. Both might lead to a derailment.

\section{Faulty Suspension \\ and -component}

Description: Faulty suspension can slide on the axle. If this situation continuous for a long period, the axle might brake due to produced thermal stress.

Faulty Suspension and -component $-25 \longrightarrow$ Unbalance (during vehicle's run)

Description: Because of a faulty suspension, axle and wheel loads may get unbalanced.

Faulty Frame $-26 \longrightarrow$ Derailment

Description: A faulty frame may lead to unbalanced wheel loads. The reduced lateral guidance of unloaded wheels might lead under bad conditions (curves, etc.) to a derailment.

Faulty Frame $-27 \longrightarrow$ Broken Axle

Description: If some elements of a faulty frame slide on an axle, the produce thermal stress and/or the material wear due to friction may lead to a breakage of the axle.

Unbalance (during vehicle's run)

Description: Unloaded wheels do not provide lateral guidance. This might lead to a derailment under bad conditions (curve, etc.).

Unbalance (during $-29 \longrightarrow$ Overriding of Buffers vehicle's run)

Description: Unbalanced stress of suspensions might cause different buffer heights. If the difference between two coupled cars or locos is too high, overriding buffers will be the consequence.

Displacement of the $-30 \rightarrow$ Unbalance (during vehicle's run)

Description: Displacement of load might cause unbalanced loads for axles respectively wheels.

Displacement of the $-31 \longrightarrow$ Violation of Clearance Gauge

Description: Displacement of load on open cars can lead to a violation of the clearance profile. 


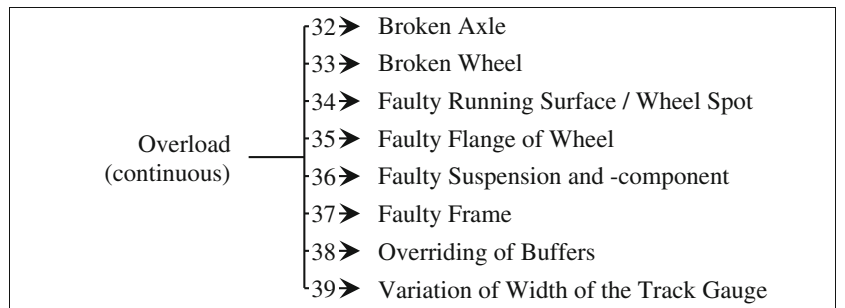

Description: If one car is overloaded continuously, its components will be highly stressed and the wear of affected components will be increased. This might lead to breakdown of one or more of such elements. Moreover the superstructure will be stressed too. Overloads can also lead to lower buffer heights and therefore cause overridings of buffers under bad conditions.

$\begin{aligned} \begin{array}{r}\text { Violation of } \\ \text { Clearance Gauge }\end{array} & 40 \rightarrow \text { Derailment } \\ 41 \rightarrow & \text { Fire on/in Board }\end{aligned}$

Description: If the clearance profile is exceeded by massive objects, a crash with applications might lead to a derailment. Straps of the load fastening, which exceed the clearance profile due to air flow, might reach the contact wire and cause a burning load.

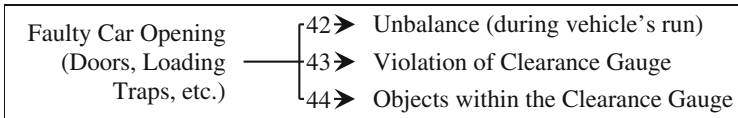

Description: Because of open car openings cargo may be lost during the run. On the one hand this might lead to unbalance load. On the other hand lost cargo might extend into the clearance profile. Moreover open doors might exceed the clearance profile.

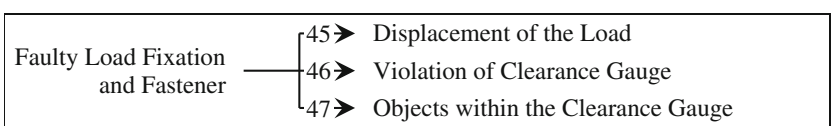

Description: Faulty fixation or insufficient fixation might lead to movement of the cargo and therefore cause unbalanced loading conditions. Moreover cargo might fall off the car and extend into the clearance profile.

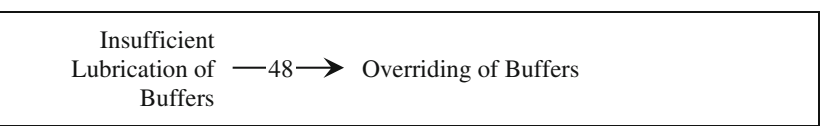

Description: If the friction between buffer discs increases due to high pressure and if there are large height displacements of touching buffers an overriding of these buffers might occur.

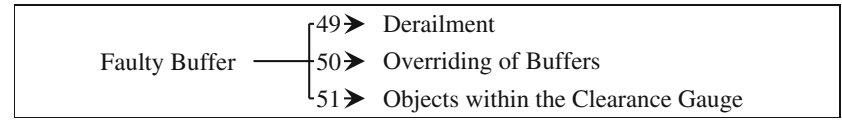

Description: If there are cracks on the buffer disc, the sliding of discs will be handicapped. Derailments or overriding buffers might be the consequence. If the buffer installation is faulty, one buffer might break, fall down and lay on the superstructure.

\section{Overriding of Buffers $-52 \longrightarrow$ Derailment}

Description: Due to an overriding of buffers it is not possible for buffer discs to slide and therefore an axle can be pressed out of the track.

$\begin{array}{r}\text { Faulty Electrical Car } \\ \text { Equipment }\end{array}-53 \rightarrow$ Fire on/in Board

Description: Faults of electrical systems on/in a vehicle might destroy the device itself and might ignite a car.

Variation of the

Width of the Track $-55 \longrightarrow$ Derailment Gauge

Description: Too large or too small track gauge might lead to a derailment.

Track Distortion $-56 \longrightarrow$ Derailment

Description: Too large track gauge might lead to a derailment.

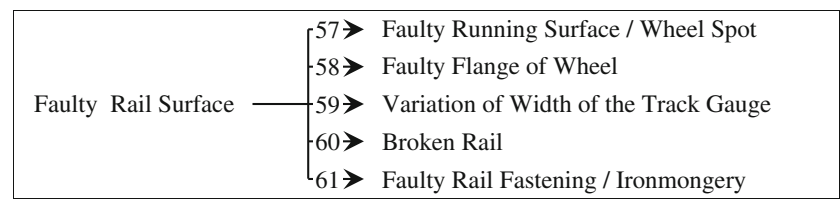

Description: If the running surface is faulty, there will be higher vertical force peaks at the rail-wheel-contact which cause higher stress for wheel and rail. 
Faults inside Rail $-62 \longrightarrow$ Broken Rail

Description: Faults inside the rail reduce the toughness of a rail and might lead to a breakage.

Worn Rail $-63 \rightarrow$ Variation of the Width of the Track Gauge

Description: High abrasive lateral attrition leads to increased wear of the inner rail surface, which means increase of track gauge width.

Worn Rail $-64 \longrightarrow$ Broken Rail

Description: High lateral and longitudinal wear cause higher stress for rails. This might lead to a breakage of a rail.

Aged Rail Material $-65 \longrightarrow$ Broken Rail

Description: Material fatigue reduces the toughness of rail and raises the risk for breakage of a rail.

Broken Rail $-66 \rightarrow$ Derailment

Description: A breakage with a damaged rail head might cause a lost of the guidance, which may lead to a derailment. If there is a vertical breakage of the rail, the rail will move lateral under stress. This might cause a derailment too.

\section{Broken Rail $\quad[7>$ Faulty Running Surface / Wheel Spot} $68 \rightarrow$ Faulty Flange of Whee

Description: Faulty heads of rails or height differences of the running surface due to vertical cracks produce force peaks at the wheel-rail-contact. This higher stress may damage the wheel.

Faulty Elastic Rail $\mathrm{Pad}-69 \longrightarrow$ Variation of the Width of the Track Gauge

Description: If the rail pad of concrete sleepers is missing or faulty, the width of the track gauge might be increased.

$\begin{array}{r}\text { Faulty Rail Fastening } \\ \text { / Ironmongery }\end{array}-70 \longrightarrow$ Variation of the Width of the Track Gauge

Description: A faulty rail mounting implies a loose connection between rail and sleepers, which might lead to an increased width of the track gauge.

Aged Timber Sleeper $-71 \longrightarrow$ Variation of the Width of the Track Gauge

Description: Old timber sleepers have reduced capability to deal with torque, which might lead to an increased width of the track gauge. $\begin{array}{r}\text { Cracks in Concrete } \\ \text { Sleeper }\end{array}-72 \longrightarrow$ Variation of the Width of the Track Gauge

Description: Longitudinal or lateral cracks of concrete sleepers lead to a reduced capability to deal with torque, which might lead to an increased width of the track gauge.

$\begin{aligned} \text { Insufficient Track } \\ \text { Bed }\end{aligned}-\left[\begin{array}{l}73> \\ 74>\text { Derailment } \\ \text { Track Distortion }\end{array}\right.$

Description: Wear of ballast causes subsidence. This might cause failures of the track. Therefore the proportion of $\mathrm{Y}$ and $\mathrm{Q}$ forces might be higher and a derailment might be the consequence. Moreover a reduced edge of ballast might reduce the lateral resistance and a track distortion might be the result.

\section{Conclusion}

For the reasons of the analysis and the validation of the supposed logical connections between different safety related fault events and states in the railways, a network of technical train monitoring components must exist. The data acquisition can be done on the vehicle-side or wayside or both together. Based upon research projects within the last years several sensor systems are now available which can be used for automated monitoring. Aim of such devices is to increase operational quality by means of prevention and therefore to guarantee a safe and reliable transport of passengers and goods. The key issue by the observations is the evaluation of the proper correlation of the collected data (allocation of fault state to vehicle number). If this practical problem can be solved, a huge database of monitored parameters and therefore of particular detailed information about important fault states and events will be available.

Under consideration of dependencies between failure states reactions on recognized failures states can be optimized. This causes a reduction of loss by prevention of secondary losses and improves the safety of railway operation. Based upon reduced secondary losses the availability of rolling stock and infrastructure should increase and therefore the efficiency of operation should also increase. Therefore a cross border recording along the European corridors is required.

Annex III of directive 2008/57/EC [5] specifies the essential requirements on interoperability which a railway system has to fulfill. Safety is one of these essential requirements and is defined by several requirements. One of them is for the design, construction or assembly, maintenance and monitoring of safety-critical components, and more particularly of the components involved in train movements.

In accordance to directive 2008/57/EC the railway companies must verify this at least in context to the conferral and 
monitoring of safety authorization based upon article 11 of directive 2004/49/EC [6] and for licensing and supervision of safety certification based upon article 10 of directive 2004/49/EC.

In the recently started European project "D-Rail - Development of the Future Rail Freight System to Reduce the Occurrences and Impact of Derailment" this methodology will be applied to identify failures in railway operation, which have to be monitored to prevent derailments.

Acknowledgement Described work was funded by the Austrian ministry of transport, technology and innovation in the frame of the program ISB - "Innovative System Railway".

Open Access This article is distributed under the terms of the Creative Commons Attribution License which permits any use, distribution and reproduction in any medium, provided the original author(s) and source are credited.

\section{References}

1. Chloupek A et al (2009) SUParBahn - Sicherheitsrelevante Überwachungsparameter im System Bahn, final report of national funded study (ISB "Innovative System Railway", Austrian Federal Ministry for Transport, Innovation and Technology)

2. Project "Integrail - intelligent integration of railway systems"; http://www.integrail.info/

3. Schöbel A (2011) Wayside train monitoring systems - an actual overview, RTR - European Rail Technology Review (Special), 2011, DVV Media Group, ISBN 978-3-7771-0426-3, ISSN 0079-9548

4. UIC, ERFA, UIP (2006) General contract of use for wagons (GCU), Edition dated 1 July 2006

5. Directive 2008/57/EG of the European Parliament and of the Council of 17 (2008) June 2008 on the interoperability of the rail system within the community (Official Journal of the European Union L 191 of 18.07 .2008$)$

6. Directive 2004/49/EC of the European Parliament and of the Council of 29 (2004) April 2004 on safety on the community's railways (Official Journal of the European Union L 164 of 30.04.2004) inclusive corrigendum (Official Journal of the European Union L 220 of 21.06.2004) 